\title{
A Correlation between Tuberculosis Infection and Coagulation Parameters (In Mile Four Hospital, Abakaliki)
}

\author{
Albert N. Eteudo ${ }^{1}$, Chuks O. Edeogu ${ }^{2}$, Igube A. Nwovu ${ }^{3}$, Chibueze C. Igwe $^{3 *}$, Clara Kela-Eke ${ }^{4}$, Simon O. Azi ${ }^{5}$, \\ Chinwe F. Ezeruigbo ${ }^{6}$ and Chituru J. Orioha6 \\ ${ }^{1}$ Department of Anatomy, Ebonyi State University, Abakaliki, Ebonyi State, Nigeria. \\ ${ }^{2}$ Faculty of Health Science and Technology, Ebonyi State University, Abakaliki, Ebonyi State. \\ ${ }^{3}$ Federal Teaching Hospital, Abakaliki, Ebonyi State, Nigeria. \\ ${ }^{4}$ University of Nigeria Teaching Hospital, Ituku-Ozalla, Enugu. \\ ${ }^{5}$ Medical Laboratory Science, Ebonyi State University, Abakaliki, Ebonyi State. \\ ${ }^{6}$ Department of Nursing Science, Ebonyi State University, Abakaliki, Ebonyi State.
}

\begin{abstract}
Background: The processes of inflammation and coagulation are known to be interconnected through a threesome mechanism (platelet activation, endogenous anticoagulant down-regulation and fibrinolysis inhibition). The present study was aimed at determining the correlation between tuberculosis and coagulation parametersamong tuberculosis patients in Mile 4 Hospital, Abakaliki.

Methods: A total of 270 venous blood samples (180 from tuberculosis patients and 90 from non-tuberculosis subjects \{as control\}) were collected and assayed for prothrombin time, activated partial thromboplastin time and platelet count using standard clotology techniques.

Results: The mean coagulation parameters - aPTT, PT and platelet count, of the patient group (46.09 $\pm 8.79 \mathrm{sec}, 15.53 \pm 3.35 \mathrm{sec}$ and $286.33 \pm 101.09 \times 109 / \mathrm{L}$ respectively) was higher than that of the control group $(30.75 \pm 4.06 \mathrm{sec}, 13.60 \pm 1.64 \mathrm{sec}$ and $238.20 \pm 92.19 \times 109 / \mathrm{L}$ respectively). Significant difference between the two groups was noted in the mean PT and aPTT $(\mathrm{p}<0.05)$. The result also showed that the mean value of the coagulation parameters, aPTT, PT $(15.93 \mathrm{sec})$ and platelet count $(292.39 \mathrm{x} 109 / \mathrm{L})$ (among the tuberculosis patients) of the male gender was higher compared to the female gender (43.56 sec, $15.26 \mathrm{sec}$ and $277.25 \mathrm{x} 109 / \mathrm{L}$ respectively). The difference in mean with respect to sex was statistically insignificant $(\mathrm{p}>0.05)$. Positive correlation exist between PT and platelet count; aPTT and platelet count and aPTT and PT.
\end{abstract}

Conclusion: It will suffice to say that there is a correlation between tuberculosis and coagulation pathway. Also, a positive relationship exist between parameters of the intrinsic (aPTT) and extrinsic (PT) coagulation pathway.

Keywords: Tuberculosis, Coagulation, Prothrombin Time, Thrompoblastin, Platelet

\section{Introduction}

Mycobacterium tuberculosis, the causative agent of tuberculosis (TB) is a bacteria with a mycolic cell wall which gives the organism its resistant property to acid[1], thus the name acid fast bacilli.Increase in the incidence of tuberculosis all over the world has been alarming. It is a significant global disease with approximately one-third of the world's population infected with the disease[1]. The risk group includes, but not limited to, the elderly, homeless, malnourished, alcoholic males, prison populations, immigrant etc. transmission is by respiratory route through droplet nuclei from infected humans. Transmission to human from susceptible animal species and their products has been reported[1,2]. Laboratory diagnosis of tuberculosis is by microscopic identification of acid fast bacilli in a Ziehl Nelson or fluorescent acid fast stained sputum smear[1].

Worthy of note, in the science of tuberculosis infection, has been the roles of various inflammatory cells, cytokines and immune affector cells which mediates in the formation of granulomatous lesions encountered in tuberculosis[1,3]. Inflammatory response to tuberculosis is initiated by macrophages through phagocytic action against the bacilli and release of cytokines. The released cytokines mobilizes other immune affector cells to the site of the infection.

Inflammation and coagulation are interlinked with the two systems interconnected through 3 main mechanisms coagulation activation, down-regulation of natural anticoagulants and inhibition of fibrinolysis[4]. Cytokines (e.g. interleukin-6 $\{$ IL-6\}) has been known to play a role in platelet production[5]. 
Several proteins called coagulation factors are involved in the process of hemostasis. When an injury occurs and bleeding begins, coagulation factors are activated in a sequential order (coagulation cascade) which terminates in clot formation[6].

Coagulation pathway is subdivided into three - extrinsic, intrinsic and common pathway. The Activated partial thromboplastin time (aPTT) test is a screening test of the intrinsic pathway. It is done primarily for the determination of the efficacy/potency of coagulation factors of the intrinsic and common pathway (prothrombin, V, VIII, IX, X, XI, XII and fibrinogen)[7]. Prothrombin time test measures factor VII, X, V prothrombin and fibrinogen (coagulation of the extrinsic and common pathway).

Laboratory tests provides invaluable information on the pathologic processes occurring in patients. Knowing the status of the inflammatory response may come in handy in formulating a treatment plan and in monitory the patient's response to therapy. Given that inflammatory and coagulation systems are closely linked, it is possible that traditional tests of coagulation are influenced by inflammation. Hence, the present study was carried out to establish the correlation between tuberculosis infection and coagulation parameters among tuberculosis patients in Mile 4 Hospital, Abakaliki.

\section{Materials \& Methods}

Study Area: The study was carried out in Mile 4 Hospital, Abakaliki. Abakaliki is the capital of present day Ebonyi State in Southeastern part of Nigeria. This hospital is the second largest hospital in the state. It is a referral hospital were special health cases like tuberculosis is taken care of.

Study Design/Population: The study population comprises of tuberculosis patients in Mile 4 hospital Abakaliki, Ebonyi State and non-tuberculosis subjects (as control) within Abakaliki metropolis. A total of 180 tuberculosis patients (test group) and 90 non-tuberculosis patients (control group) were recruited in this study.

Ethical Consideration: The consent of The Medical Director, Mile 4 hospital, Abakaliki was sort and obtained. Also, the aim of the study was made known to each subject and they gave their consent before been enrolled in the study.

Sample Collection: Venous blood was obtained from each subject and transferred into a container, containing trisodium citrate anticoagulant (in a ratio of $0.25 \mathrm{ml}$ of anticoagulant against $2.25 \mathrm{ml}$ of blood sample). Another $2 \mathrm{ml}$ of blood was added into an EDTA container for platelet count. Each container was properly labeled to avoid sample mix up. All samples were analyzed within two hours of collection.

\section{Laboratory Analysis:}

Prothrombin Time Test: this was determined using Calcium thromboplastin test kit (Plasmascann ${ }^{\circledR}$, Quinica Clinica Aplicada (QCA), South Africa) as documented by Chessbrough[7]. The principle of the test is based on the ability of citrated blood to clot in the presence of tissue thromboplastin and $\mathrm{CaCl}_{2}$. The citrated blood collected from each subject (containing triSodium citrate and venous blood at a ratio of 9:1) was properly mixed and centrifuged at $3000 \mathrm{rpm}$ for 15 minutes and the plasma separated into a test tube.The prothrombin time (PT) reagent was reconstituted with $10 \mathrm{ml}$ of distilled water, allowed to stand for 10 minutes for proper reagent homogenization and then pre-warmed in a water bath at $37^{\circ} \mathrm{C}$ for 15 minutes. The citrated plasma was pre-warmed at $37^{\circ} \mathrm{C}$ and $0.1 \mathrm{ml}$ patient citrated plasma (prewarmed at $37^{\circ} \mathrm{C}$ ) was added to $0.2 \mathrm{ml}$ tissue thromboplastin reagent with calcium (prewarmed at $37^{\circ} \mathrm{C}$ ), the water was stirred immediately and the time for clot formation was noted and recorded.

Activated Partial Thromboplastin Time Test (aPTT): this is the functional determinant of the intrinsic and common pathway. The principle of this test depends on the ability of a citrated blood to clot in the presence of a reagent containing platelet phospholipid and $\mathrm{CaCl}_{2}$. The time interval between the additions of the reagents and clot formation is called the activated partial thromboplastin time. It is measured in seconds. A sufficient quantity of calcium chloride reagent was pre-warmed to $37^{\circ} \mathrm{C}$ and 0.1 $\mathrm{ml}$ of the test sample (plasma) and control was pipetted into the appropriate labeled test tube using automatic pipette. To each test tube, $0.1 \mathrm{~mL}$ of partial thromboplastin reagent was added using the automatic pipette and then mixed carefully.Sample-partial thromboplastin reagent mixture was allowed to pre-warm to $37^{\circ} \mathrm{C}$ for 5 minutes. $0.1 \mathrm{ml}$ pre-warmed calcium chloride reagent was then pipetted into the test tube containing sample-partial thromboplastin reagent mixture using the automatic pipette. When the calcium chloride reagent was dispensed, the timer was started simultaneously.The time taken for clot formation was noted and recorded[7].

Platelets Count: Blood was diluted 1 in 20 in a filtered solution of $1 \%$ ammonium oxalate reagent which lyses the red cells. Platelets were counted using an improved Neubauer counting chamber and the number of platelets per blood calculated. Platelet was measured in $\times 10^{9} /$ liter[7].

Statistical Analysis: All data were analyzed using SPSS (Statistical Package for Social Sciences) version 20.0. Descriptive statistics were computed for all relevant data. Pearson product-moment correlation technique was used to 
correlate the changes in Platelet, Prothrombin Time and activated Partial Thromboplastin Time for statistical significance.

\section{Result}

A total of 270 venous blood samples were used. 180 (108 male and 72 female) of the samples were from tuberculosis patients while 90 were from non-tuberculosis individuals (control). The mean age of the patients and control group was $36.333 \pm 11.992$ and $36.533 \pm 11.420$ respectively.

The mean value of the coagulation parameters (among the tuberculosis patients) in relation to gender was presented graphically in fig. 1. Male gender had a higher aPTT, PT and platelet count (47.78 sec, $15.93 \mathrm{sec}$ and $292.39 \times 10^{9} / \mathrm{L}$ respectively) compared to the female gender (43.56 sec, $15.26 \mathrm{sec}$ and $277.25 \times 10^{9} / \mathrm{L}$ respectively)
Table 1 showed the Mean value, Standard Deviation and Independent $\mathrm{t}$ test value of the coagulation parameters. The mean coagulation parameters - aPTT, PT and platelet count, of the patient $(46.09 \pm 8.79 \mathrm{sec}, 15.53 \pm 3.35 \mathrm{sec}$ and $286.33 \pm 101.09 \times 10^{9} / \mathrm{L}$ respectively) was higher than that of the control group $(30.75 \pm 4.06 \mathrm{sec}, 13.60 \pm 1.64 \mathrm{sec}$ and $238.20 \pm 92.19 \times 10^{9} / \mathrm{L}$ respectively). Significant difference between the two groups was noted in the mean PT and aPTT $(\mathrm{p}<0.05)$.

Table 2 showed the correlation analysis of the coagulation parameters (Platelet count, PT and aPTT). Positive correlation exist between PT and platelet count $(\mathrm{r}=0.006$; $\mathrm{p}=0.976)$, aPTT and platelet count $(\mathrm{r}=0.171 ; \mathrm{p}=0.366)$ and aPTT and PT $(0.103 ; \mathrm{p}=0.303)$.

Table 1: Mean value, Standard Deviation and Independent t test value of the coagulation parameters of the patient and control group.

\begin{tabular}{|l|c|c|c|}
\multirow{2}{*}{ Parameter } & Patient & Control group & P- value \\
\cline { 2 - 4 } & Mean \pm Standard Deviation & Mean \pm Standard Deviation & \\
\hline Age (years) & $36.33 \pm 11.99$ & $36.53 \pm 11.42$ & 0.9580 \\
Platelet count $\left(\times 10^{9} / \mathrm{l}\right)$ & $286.33 \pm 101.09$ & $238.20 \pm 92.19$ & 0.1290 \\
*PT (seconds) & $15.53 \pm 3.35$ & $13.60 \pm 1.64$ & 0.0130 \\
*aPTT (seconds) & $46.09 \pm 8.79$ & $30.75 \pm 4.06$ & 0.0003 \\
\hline
\end{tabular}

$P T=$ Prothrombin time; aPTT = Activated Partial Thromboplastin Time; *statistically significant at 0.05 level of significance.

Table 2: Correlation analysis of the coagulation parameters of the patient and control group.

\begin{tabular}{|c|c|c|}
\hline Parameters & P-value & r-value \\
\hline $\mathrm{PT}_{\text {patient }}$ - Platelet count & 0.976 & 0.006 \\
\hline aPTT $_{\text {patient }}$ - Platelet count & 0.366 & 0.171 \\
\hline $\mathrm{aPTT}_{\text {patient }}-\mathrm{PT}_{\text {patient }}$ & 0.103 & 0.303 \\
\hline
\end{tabular}

$P T=$ Prothrombin time, aPTT = activated Partial Thromboplastin Time, $P$-value $=$ probability value for the degree of significance, $r$-value $=$ correlation coefficient

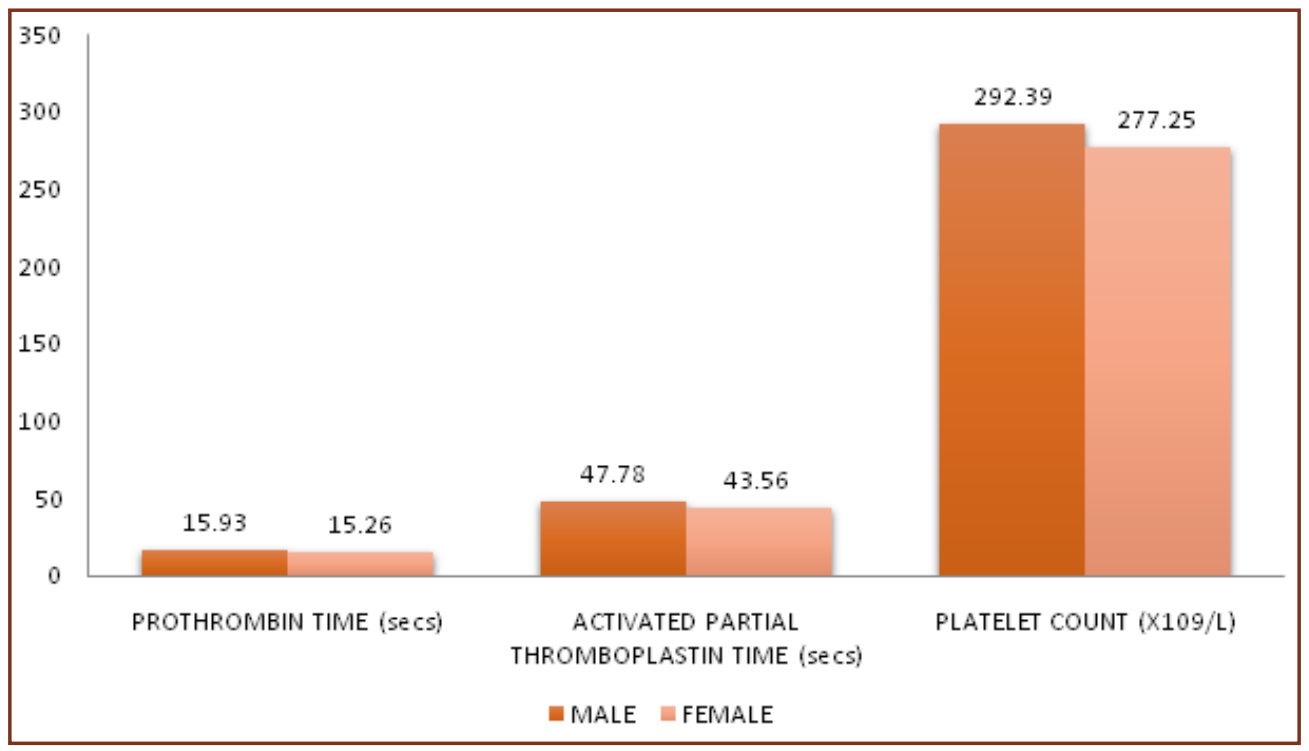

Fig. 1: The mean values of the coagulation of the patient group with respect gender. 


\section{Discussion}

A previous study by Can and colleaguesreported that inflammation increases coagulation parameters (prothrombin time, activated partial thromboplastin time and platelet count)[8]. However, the mean platelet count of the patient was insignificantly higher than that of the control group.

Increased prothrombin time reported in this study has been reported elsewhere among patients with pulmonary tuberculosis[8,9]. The authors above also stated that cytokines and mediators emerging from a tuberculosis lesion are considered to prolong prothrombin time.

There is increased platelet production amongst the patient when compared with the control group. Reports elsewhere has shown that cytokines are increased in inflammatory reactions and also, these cytokines (mainly IL-6) increases platelet production[3,5]. Also, inflammatory mediators (e.g. bacteria endotoxins, thromboxane A2 etc) activate platelet. The production and activation of platelet leads to the activation of secondary hemostatic pathway (formation of thrombin). The interaction between activated platelets and sub endothelial cells stimulates the adhesion and the release of cytokine Interleukin-1 $\beta$ (IL-1 $\beta$ ) by inflammatory leukocytes, which enhances the adhesion properties of endothelial cells[11]. The activated endothelial cells express Tissue thromboplastin that, linked to FVIIa, activates coagulation pathway[12].

Analysis of the mean values of the patients PT, aPTT and platelet count with respect to gender showed that the PT, aPPT and platelet count of the male patient were higher than that of the female patients. The difference in mean of coagulation parameters was not dependent on gender $(p>0.05)$. The reason for the increase in the coagulation parameters of male as compared to female could be attributed to gender distribution in the sample size which showed a male to female ratio of 1.5. The high population of male being infected with tuberculosis in the present study may be due to factors like life style (e.g. alcohol and smoking factor), environmental factors like working in overcrowded environments with poor ventilation (in the presence of TB patients) etc.

The result of correlation of the coagulation parameters showed a weak but insignificant positive correlation between PT and platelet count; aPTT and platelet count, and aPTT and PT among tuberculosis patients. Similar correlation between aPTT, PT and platelet count has been reported by Okoroiwu and co-researchers among HIV patients in Owerri, Southeastern Nigeria[13].
Based on the findings of the present study, it will suffice to say that there is a correlation between tuberculosis and coagulation pathway. Also, a positive relationship exist between parameters of the intrinsic (aPTT) and extrinsic (PT) coagulation pathway.

\section{Conflicts of Interest}

The authors declare that they have no conflicting interests.

\section{Acknowledgement}

The authors wish to say a big thank you to all staff of Mile 4 hospital, Abakaliki, especially those in the Laboratory Services Unit, for their assistance during sample collection. Also, the authors will wish to acknowledgement all the subjects who consented positively to this study.

\section{References}

1. Prescott, L. M., Harley, J. P. and Klein, D. A. Microbiology. 6th edition. McGraw Hill Higher Education. New York. 2005; p.990.

2. McPherson, R.A. and Pincus, M.R. Henry's Clinical Diagnosis and Management by Laboratory Methods 22nd Edition. 1600 John F. Kennedy Blvd, Site 1800, Philadelphia, PA. 2011; pp.1-1508.

3. Bost, T.W., Riches, D.W. and Schumacher, B. Alveolar macrophages from patients with beryllium disease and sarcoidosis express increased levels of mRNA for tumor necrosis factor alpha and interleukin-1 beta. American Journal of Respiratory Cell Molecular Biology. 20O4; $10: 1506-13$

4. Walker, C.P.R. and Royston, D. Thrombin generation and its inhibition: a review of the scientific basis and mechanism of action of anticoagulant therapies. $\mathrm{Br} \mathrm{J}$ Anaesth. 2002;88:848-63.

5. Hill, R.J., Warren, M.K. and Levin, J. Stimulation of thrombopoiesis in mice by human recombinant interleukin-6. Journal of Clinical Investigation. 2000;85:1242-7.

6. Hoffman, A.V., Moss, P.A.H. and Pettit, J.E. Essential Haematology. 5th Ed. Blackwell Publishing Inc., 350 Main Street, Malden, Massachusetts, USA. 2006; pp.1-380.

7. Cheesbrough, M. Haematological Tests: District Laboratory Practice Tropical Countries, Part 2, 2ndedition, Cambridge University Press, United Kingdom. 2006; pp. 272-330.

8. Can D., Aliye, S. and Oya, O. Coagulation parameters in inflammatory bowel disease. Int. J. Clin Exp. Med.2014; 7(5):1442-1448

9. Kartaloglu, Z., Bilgic, H., Aydilek, R., Cerrahoglu, K., Koylu, R. and Kunter, E. Platelets in pulmonary and pleural tuberculosis. Tuberculosis and Lung Disease. 2005;76(2):30.

10. Kartaloglu, Z., Cerrahoglu, K., Okutan, O., Ozturk, A. and Aydilek, R. Parameters of Blood Coagulation in Patients 
with Pulmonary Tuberculosis. The Internet Journal of Internal Medicine. 2002; 2:2.

11. Ward, P.A. and Cochrane, C.G. Bound complement and immunologic injury of blood vessels. J Exp Med. 1965;121:215-234.

12. Graham, R.C., Ebert, R.H., Ratnoff, O.D. and Moses, J.Pathogenesis of inflammation. II. In vivo observations of the inflammatorv effects of activated Hageman factor and bradykinin. J Exp Med. 1965; 121:807-820, 1965.

13. Okoroiwu, I.L., Amadi, U., Obeagu, E.I., Anode, A. and Udokwu, E.I. The Correlation of Values of $\mathrm{Cd} 4$ Count, Platelet, Pt, Aptt, Fibrinogen and Factor VIII Concentrations among HIV Positive Patients in FMC Owerri. Journal of Dental and Medical Sciences. 2014;13(9):94-101.

*Corresponding author:

Igwe, Chibueze C., Federal Teaching Hospital, Abakaliki, Ebonyi State, Nigeria.

Email: scientistigwe@gmail.com 